\title{
14. Nähe auf Distanz: Bleiben die Beziehungen zwischen älteren Eltern und ihren erwachsenen Kindern trotz wachsender Wohnentfernungen gut?
}

\author{
Katharina Mahne \& Oliver Huxhold
}

\section{Kernaussagen}

Personen im mittleren Alter haben 2014 seltener Kinder, aber häufiger noch lebende Eltern als 1996: Die 42- bis 47-Jährigen hatten im Jahr 1996 zu 87,2 Prozent Kinder, im Jahr 2014 sind es nur noch knapp 80 Prozent. Hingegen ist in derselben Altersgruppe der Anteil derjenigen, die noch mindestens ein lebendes Elternteil haben, von 81,2 Prozent im Jahr 1996 auf 91,1 Prozent im Jahr 2014 gestiegen.

Die Wohnentfernung zwischen Eltern und ihren erwachsenen Kindern vergrößert sich im sozialen Wandel stetig: Während die erwachsenen Kinder im Jahr 1996 für 38,4 Prozent der Eltern in der Nachbarschaft oder im gleichen Ort lebten, trifft dies im Jahr 2014 nur noch auf etwa ein Viertel (25,8 Prozent) zu. Von wachsenden Wohnentfernungen sind jüngere Eltern stärker betroffen als ältere Eltern.

Insbesondere hochgebildete Eltern haben weiter entfernt wohnende erwachsene Kinder: Im Jahr 2014 leben ungefähr ein Drittel der erwachsenen Kinder von Eltern mit niedriger Bildung in der Nachbarschaft oder im gleichen Ort (35,6 Prozent). Bei den hochgebildeten Eltern tun dies nur 19,9 Prozent.

Die Kontakthäufigkeit und die Beziehungsenge zwischen Eltern und erwachsenen Kindern bleiben stabil hoch: Im gesamten Zeitraum zwischen 1996 und 2014 haben mehr als 78 Prozent der Eltern mindestens wöchentlich Kontakt und mehr als 88 Prozent der Eltern haben eine enge oder sehr enge Beziehung zu ihren erwachsenen Kindern. Mütter haben kontaktintensivere und emotional engere Beziehungen als Väter.

Gefühle wie Wut und Ärger sind in den Beziehungen zwischen Eltern und ihren erwachsenen Kindern selten: Gut drei Viertel der Eltern (77,1 Prozent) empfinden im Jahr 2014 nie oder nur selten Wut und Ärger gegenüber ihren Kindern. Etwa ein Fünftel (20,3 Prozent) berichtet davon, manchmal Wut und Ärger zu empfinden. Mütter und jüngere Eltern berichten häufiger von Gefühlen wie Wut und Ärger. 


\subsection{Einleitung}

Familiale Beziehungen sind eine wesentliche Quelle für soziale Integration, Lebensqualität und Wohlbefinden über den gesamten Lebenslauf hinweg. Die Bindungen zu den eigenen Kindern und Enkeln gehören dabei zu den engsten sozialen Beziehungen älter werdender Menschen (Rossi \& Rossi 1990). Sie vermitteln Gefühle von Zugehörigkeit, Intimität und Sinnhaftigkeit und sind ein wesentlicher Bestandteil der Alltagsgestaltung. Aber auch Gefühle von Wut und Ärger sind Teil dieser Beziehungen.

Der demografische und soziale Wandel wirkt auf familiale Strukturen und die Ausgestaltung familialer Beziehungen. Die in Deutschland anhaltend niedrige Geburtenrate führt einerseits zu schmaleren familialen Netzwerken - zum Beispiel gibt es weniger Geschwister innerhalb einer Generation. Deutschland gilt zudem als eines der europäischen Länder mit den höchsten Anteilen an Kinderlosen. Andererseits sind die Lebenssituationen in vielen Fällen vom gleichzeitigen Vorhandensein mehrerer familialer Generationen gekennzeichnet. Aufgrund der steigenden Lebenserwartung stellen Drei-Generationen-Konstellationen innerhalb einer Familie heute den Regelfall dar, auch Vier-Generationen-Konstellationen sind keine Seltenheit. Großelternschaft ist heute für viele Ältere eine $\mathrm{zu}$ erwartende und über einen langen Zeitraum gestaltbare Altersrolle.

Für die Beschreibung von familialen Strukturen hat sich der Begriff der ,multilokalen Mehrgenerationenfamilie ${ }^{c}$ (Bertram 2000) etabliert. Hierin drücken sich neben den generationalen auch räumliche Strukturen von heutigen Familien aus - Eltern und erwachsene Kinder führen heute häufiger getrennte Haushalte (Peuckert 2012) und wohnen immer öfter weit voneinander entfernt (Mahne \& Motel-Klingebiel 2010). Da familiale Generationenbeziehungen eine wichtige Stütze bei der Alltagsbewältigung älterer Menschen darstellen, werden Kinderlosigkeit sowie wachsende Wohnentfernungen in jüngeren Kohorten oftmals als Gefahr für den familialen Zusammenhalt und die Aufrechterhaltung von Lebensqualität Älterer diskutiert. Die Beschreibung von ,Nähe auf Dis- tanz' (Rosenmayr \& Köckeis 1963) trifft allerdings auf die tatsächlich gelebten Generationenbeziehungen besser zu. Große Entfernungen müssen nicht zwangsläufig mit einer schlechten Beziehungsqualität einhergehen, wenngleich räumliche Nähe eine Voraussetzung für persönlichen Kontakt und gegenseitige Hilfen im Alltag ist. Zudem werden Freunde in den Netzwerken Älterer bedeutsamer und übernehmen nicht selten Unterstützungsfunktionen (Huxhold, Mahne, \& Naumann 2010) (vgl. auch Kapitel 17). Die strukturellen, affektiven und funktionalen Aspekte von Generationenbeziehungen sind also in unterschiedlicher Weise Veränderungen unterworfen. Zusätzlich kann sich dieser Wandel für bestimmte Gruppen unterscheiden - zum Beispiel zwischen Älteren und Jüngeren, Männern und Frauen oder höher und niedriger gebildeten Menschen.

Das Aufschieben von Geburten in spätere Lebensphasen, geringe Kinderzahlen oder Kinderlosigkeit betreffen in Deutschland vorrangig jüngere Kohorten und höher gebildete Frauen. Altern in kleinen familialen Netzen oder das Ausbleiben von Elternschaft (und Großelternschaft) und damit das Abbrechen der Generationenlinie betrifft sie also in besonderer Weise. Das Geschlecht wird weiterhin als wesentlicher Faktor für die Ausgestaltung und Qualität familialer Beziehungen angesehen. Frauen unterhalten in der Regel - möglicherweise aufgrund von spezifisch erlernten Kompetenzen oder Geschlechterrollenerwartungen - kontaktintensivere und emotional engere Beziehungen als Männer. Im Zuge eines allgemeinen Aufweichens von Geschlechterrollen wäre es jedoch möglich, dass sich in jüngeren Kohorten beispielsweise Väter in den Beziehungen zu ihren Kindern weniger stark von den Müttern unterscheiden als noch vor zwei Jahrzehnten. Im Bereich der Beziehungsstrukturen und -gestaltung sind weiterhin Unterschiede zwischen Bildungsgruppen anzunehmen. Höher Gebildeten wird im Vergleich zu niedriger Gebildeten oftmals eine schwächere Orientierung auf die Familie zugeschrieben. Allerdings verfügen höher gebildete Menschen über größere materielle und soziale Ressourcen, 
um Familienbeziehungen zu gestalten oder Probleme zu überwinden. Familien von hochgebildeten Eltern und Kindern sind zudem von einer größeren räumlichen Mobilität betroffen - und damit von weiter gestreuten familialen Netzen als niedriger Gebildete.

Negative Beziehungsaspekte wie Ärger und Wut gegenüber Familienmitgliedern werden bislang seltener untersucht als positive Inhalte und unterstützende Funktionen familialer Bindungen. Mit dem Deutschen Alterssurvey (DEAS) ist die Analyse dieser Aspekte erstmals 2014 für die zweite Lebenshälfte möglich. Konflikte mit den Kindern sind möglicherweise in bestimmten Familienphasen virulenter als in anderen. Zudem ist aufgrund der Unterschiede bei der Beziehungsgestaltung zwischen Müttern und Vätern ein unterschiedliches Ausmaß an Wut und Ärger gegenüber den Kindern zu erwarten wenngleich noch unklar ist, wer von Konflikten häufiger berichtet. So haben Mütter in der Regel häufiger und engeren Kontakt zu den Kindern. Dies kann einerseits ein größeres Verständnis bedingen, anderseits mag es aber auch mehr Gelegenheiten für Konflikte mit sich bringen.

Im vorliegenden Kapitel werden die folgenden Fragen untersucht:

1. Wie wandeln sich die Strukturen von familialen Generationenbeziehungen?

2. Wächst die Wohnentfernung zwischen Eltern und erwachsenen Kindern weiter an?

3. Bleiben Kontakthäufigkeit und emotionale Nähe zwischen Eltern und erwachsenen Kindern stabil hoch?

4. Wie häufig sind Gefühle von Ärger und Wut in den Beziehungen zu erwachsenen Kindern?

\subsection{Datengrundlage ${ }^{1}$}

Daten. Für die Analyse der Generationenstrukturen wurde die gesamte Stichprobe genutzt - das heißt alle Befragten im Alter von 40 bis 85 Jahren. Analysen zur konkreten Beziehungsgestaltung beschränken sich auf Personen, die über die jeweils interessierenden Beziehungen auch tatsächlich verfügen. Für alle untersuchten Indikatoren liegen Informationen durchgängig seit 1996 vor. Einzige Ausnahme bildet die Häufigkeit des Empfindens von Wut und Ärger gegenüber den Kindern - diese Frage wurde 2014 zum ersten Mal gestellt. Für die Analyse der Beziehungsgestaltung (Wohnentfernung, Kontakthäufigkeit, Beziehungsenge, Wut und Ärger) wurden jeweils alle erwachsenen, mindestens 18-jährigen Kinder außerhalb des Haushalts der befragten Person betrachtet. Diese Einschränkung erfolgt, da beispielsweise Kontakte innerhalb eines Haushalts in der Regel

1 Die Daten des DEAS können für wissenschaftliche Zwecke kostenlos beim Forschungsdatenzentrum des DZA (www.fdz-dza.de) bezogen werden. häufig sind und zudem anderen Mustern folgen als Kontakte über die Haushaltsgrenze hinaus.

Generationenstrukturen. Bei der Analyse der Existenz von lebenden Vertreterinnen und Vertretern verschiedener familialer Generationen (beziehungsweise bei Kinderlosigkeit) wurde auf eine Unterscheidung zwischen leiblichen und nichtleiblichen Verwandten verzichtet. In diesem Kontext ist es bedeutsamer, die Existenz und damit die aktuelle Voraussetzung für eine Beziehungsgestaltung abzubilden - unabhängig vom Zustandekommen der Beziehung.

Wohnentfernung. Die Wohnentfernung bezieht sich auf mittlere Angaben zu allen mindestens 18-jährigen Kindern außerhalb des elterlichen Haushalts. Zusätzlich verwendet wurde eine Variable, die die Wohnentfernung zum jeweils nächstwohnenden, mindestens 18-jährigen Kind außerhalb des elterlichen Haushalts anzeigt. Die fünf Antwortvorgaben für beide Angaben wurden zur besseren Veranschaulichung wie folgt zusammengefasst: Wohnentfernung: 1 = ,in der Nachbarschaft/im 
gleichen Ort; 2 = , anderer Ort, aber innerhalb von zwei Stunden erreichbar'; $3=$,weiter weg' (,weiter entfernt in Deutschland'/,weiter entfernt im Ausland').

Kontakthäufigkeit. Bei der Erfassung der Kontakthäufigkeit wurde nicht zwischen persönlichen Kontakten und beispielweise Telefonaten unterschieden. Die Angaben zur Kontakthäufigkeit beziehen sich auf mittlere Angaben zu allen mindestens 18-jährigen Kindern außerhalb des elterlichen Haushalts. Die Antwortvorgaben wurden wie folgt zusammengefasst: $1=$ ,seltener' (,nie'/,seltener $/$,mehrmals im Jahr'); $2=$,mindestens monatlich (,ein- bis dreimal im Monat'); 3 = ,mindestens wöchentlich' (,mehrmals pro Woche'/,täglich').

Beziehungsenge. Erfragt wird hier die Enge der Verbundenheit. Die Beziehungsenge bezieht sich auf mittlere Angaben zu allen mindestens 18-jährigen Kindern außerhalb des elterlichen Haushalts. Die Antwortvorgaben wurden wie folgt zusammengefasst: 1 = ,eng' (,sehr eng'/ ,eng $\left.{ }^{c}\right) ; 2=$,mittel'; $3=$,nicht eng (,weniger eng'/ überhaupt nicht eng').

Wut und Ärger. Die Häufigkeit von Wut und Ärger bezieht sich auf mittlere Angaben zu allen mindestens 18-jährigen Kindern außerhalb des elterlichen Haushalts. Die Antwortvorgaben wurden wie folgt zusammengefasst: $1=$,eher selten $^{`}\left(\right.$,nie $/$, selten $\left.^{`}\right) ; 2=$, manchmal'; $3=$,eher häufig' (,häufig'/,immer').

Gruppierungsvariablen. Zur Prüfung von Altersunterschieden wurden - analog zur Schichtung der Stichprobe - drei Altersgruppen verwendet: 40 bis 54 Jahre, 55 bis 69 Jahre und 70 bis 85 Jahre. Alters- beziehungsweise Kohortenunterschiede im Wandel wurden anhand von SechsJahres-Altersgruppen untersucht, um Überschneidungen zwischen Erhebungszeitpunkten und Altersgruppen zu vermeiden ( 42 bis 47 Jahre, 48 bis 53 Jahre, 54 bis 59 Jahre, 60 bis 65 Jahre, 66 bis 71 Jahre, 72 bis 77 Jahre, 78 bis 83 Jahre). Weiterhin wurden Unterschiede nach Geschlecht und Landesteil (Ost-/Westdeutschland) geprüft. Zur Untersuchung von Bildungsunterschieden wurden - basierend auf einer reduzierten ISCED-Klassifizierung - drei Bildungsgruppen unterschieden: Personen mit niedriger, mittlerer und hoher Bildung (vgl. Kapitel 2).

Analyse. In den Abbildungen und im Text sind gewichtete prozentuale Verteilungen angegeben. Zur Testung von signifikanten Gruppenunterschieden wurden binäre und ordinale logistische sowie lineare Regressionen gerechnet. Hierbei wurde für die Stratifizierungsvariablen Altersgruppe, Geschlecht und Region (Ost-/ Westdeutschland) kontrolliert. Das genaue Vorgehen ist in Kapitel 2 beschrieben.

\subsection{Wandel der Strukturen von familialen Generationenbeziehungen}

Im Folgenden werden die Ergebnisse zu den Fragen präsentiert. Zunächst geht es um strukturelle Aspekte von Generationenbeziehungen wie das Vorhandensein bestimmter Familienmitglieder (zum Beispiel Eltern oder Kinder) und die Wohnentfernung zwischen Eltern und ihren erwachsenen Kindern. Dann werden spezifische Aspekte der Beziehungsgestaltung wie Kontakthäufigkeit, Beziehungsenge sowie das Empfinden von Wut und Ärger näher betrachtet.
Der DEAS hat bereits für die Jahre 1996 bis 2008 gezeigt, dass die intergenerationalen Strukturen im Wandel begriffen sind (Mahne \& Motel-Klingebiel 2010). Veränderungen bei der Existenz lebender Familienmitglieder werden hier über den Gesamtzeitraum von 1996 bis 2014 beschrieben, denn der soziale Wandel zeigt sich oftmals erst über eine längere Zeitspanne deutlich. 


\section{Personen im mittleren Alter haben 2014 seltener Kinder, aber häufiger noch lebende Eltern als 1996.}

Für die Existenz familialer Generationenbeziehungen, die aus Sicht der Befragten älter sind also beispielsweise die eigenen Eltern - sind in allen Altersgruppen zwischen 42 und 71 Jahren über den gesamten Zeitraum von 1996 bis 2014 die Anteile gewachsen (Abbildung 14-1a). Über 90 Prozent der 42- bis 47-Jährigen haben im Jahr 2014 noch lebende Eltern (91,1 Prozent), im Jahr 1996 sind es noch zehn Prozent weniger gewesen (81,2 Prozent). In der Altersgruppe der 60- bis 65-Jährigen ist es im Jahr 2014 ein Drittel (33,3 Prozent), 18 Jahre früher nur ein Fünftel (20,0 Prozent). Für die 66- bis 71-Jährigen hat sich der Anteil derjenigen mit mindestens einem lebenden Elternteil seit 1996 von 6,1 Prozent auf 12,4 Prozent im Jahr 2014 sogar verdoppelt. Eine ähnliche Entwicklung zeigt sich für das Vorhandensein mindestens eines lebenden Großelternteils (ohne Abbildung) - aber naturgemäß nur für die jüngsten Altersgruppen. Für die 42- bis 47-Jährigen hat sich der Anteil mit Großeltern seit 1996 etwa verdoppelt: Im Jahr 2014 hat etwa jede zehnte Person (10,4 Prozent) in dieser Altersgruppe lebende Großeltern, im Jahr 1996 waren es noch 5,3 Prozent.

Betrachtet man nun aus der Sicht der Befragten die nachfolgenden Generationen in der Familie - also Kinder, Enkel und Urenkel - zei- gen sich folgende Veränderungen: Für die 42- bis 53-Jährigen sind im Vergleich von 2014 zu 1996 kleinere Anteile von Personen mit Kindern zu beobachten. Bei den beiden nächstälteren Gruppen (54 bis 65 Jahre) zeigen sich erst ab 2002 geringere Anteile von Eltern (Abbildung 14-1b). Für die 66- bis 77-Jährigen hingegen sind im sozialen Wandel höhere Anteile von Personen mit Kindern zu beobachten. In der ältesten hier betrachteten Altersgruppe (78 bis 83 Jahre) zeigen sich keine Veränderungen über die Zeit. Die dargestellten Anteile von Personen mit Kindern geben gleichzeitig Aufschluss über die Entwicklung von Kinderlosigkeit. Für die kohortenspezifische Entwicklung des Anteils von Personen mit beziehungsweise ohne Kinder über die Zeit sind unterschiedliche Faktoren verantwortlich. Insbesondere in der jüngsten Altersgruppe (42 bis 47 Jahre), die sich (noch) in der Familiengründungsphase befindet, sind Entscheidungsund Opportunitätskonflikte in Bezug auf die Vereinbarkeit von Familie und Beruf virulent. Offenbar führen diese immer häufiger zu einer - mehr oder weniger bewussten - Entscheidung gegen Kinder. Allerdings kann für die jüngste Altersgruppe noch nicht mit Sicherheit angenommen werden, dass endgültig keine Geburten mehr erfolgen und alle diese Menschen dauerhaft kinderlos bleiben. Möglicherweise fällt der tatsächliche Anteil Kinderloser in diesen Geburtskohorten geringer aus, weil sich hier auch ein Aufschieben von Geburten in spätere Lebensphasen zeigt. 
Abbildung 14-1: Existenz lebender Eltern, Kinder und Enkelkinder, nach Alter, in den Jahren 1996, 2002, 2008 und 2014 (in Prozent)

a) Eltern

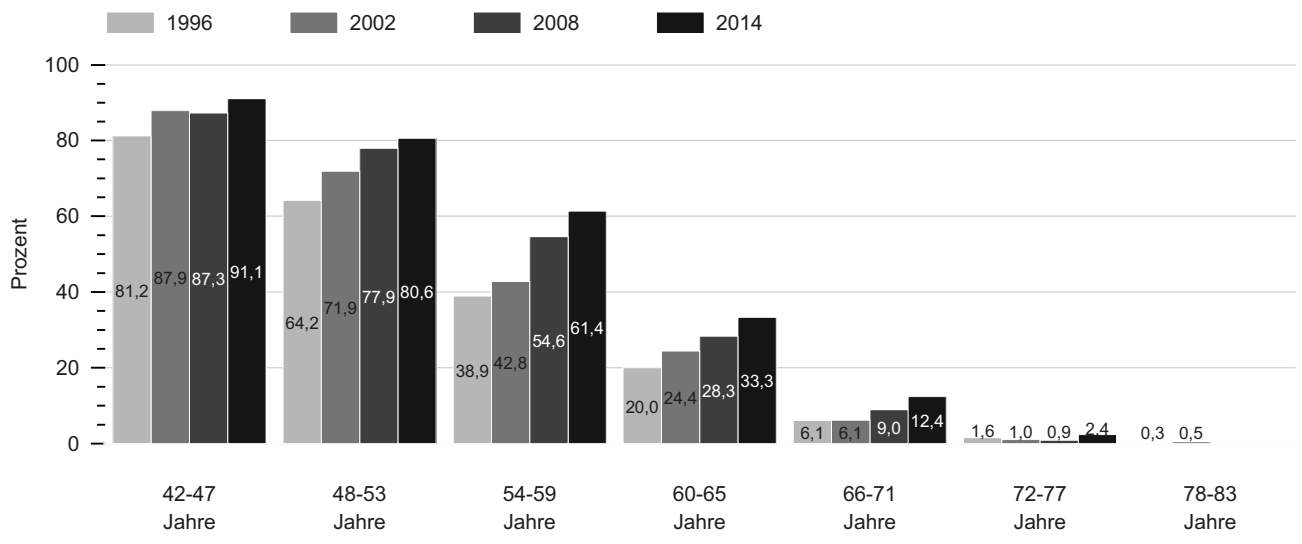

b) Kinder

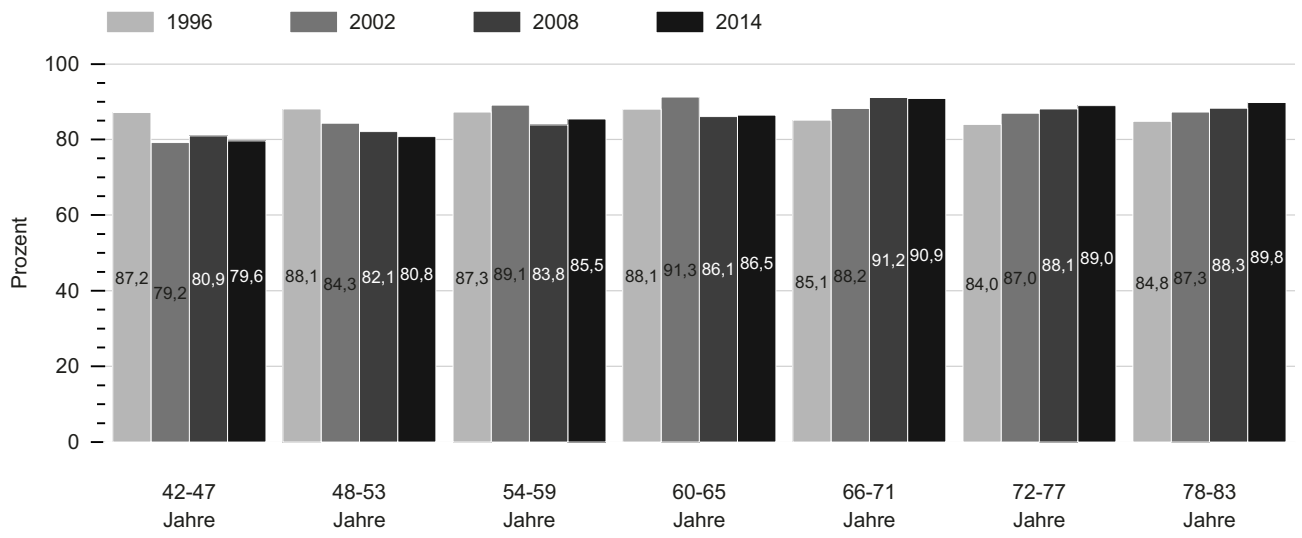


c) Enkelkinder

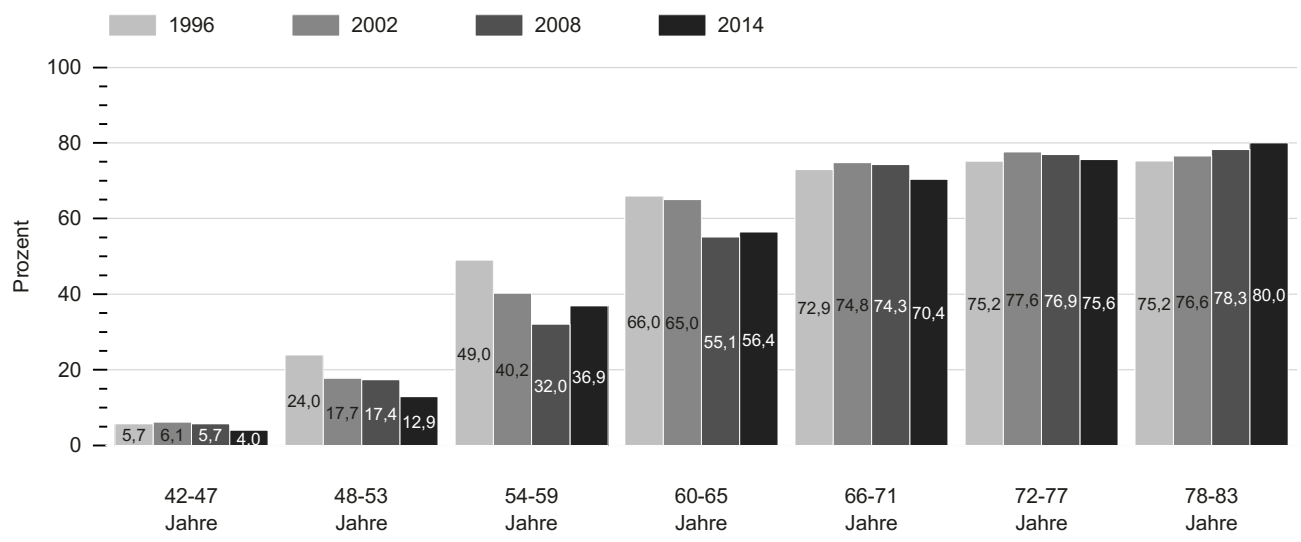

Quelle: DEAS 1996 ( $n=4.339$ für Eltern; $n=4.807$ für Kinder; $n=4.806$ für Enkelkinder), 2002 ( $n=2.691$ für Eltern; $n=3.074$ für Kinder; $n=3.011$ für Enkelkinder), 2008 ( $n=5.382$ für Eltern; $n=6.185$ für Kinder; $n=6.154$ für Enkelkinder), 2014 ( $n=5.172$ für Eltern; $n=5.982$ für Kinder; $n=5.954$ für Enkelkinder), gewichtet, gerundete Angaben; $(p<, 05)$.

a) Signifikante Unterschiede zwischen 1996 und 2014 für alle Altersgruppen außer für die über 72-Jährigen. b) Signifikante Unterschiede zwischen 1996 und 2014 für alle Altersgruppen außer für die 54- bis 59- sowie 60- bis 65-Jährigen. c) Signifikante Unterschiede zwischen 1996 und 2014 nur für die Altersgruppen 48 bis 53, 54 bis 69, sowie 60 bis 65 Jahre.

Allerdings ist anzunehmen, dass in den Altersgruppen zwischen 48 und 65 Jahren die Familiengründung zum größten Teil bereits abgeschlossen ist und nur ein sehr geringer Anteil zu einem späteren Zeitpunkt noch den Übergang zur Elternschaft erlebt. Insofern lassen die Ergebnisse darauf schließen, dass nachfolgende Kohorten zu einem größeren Anteil dauerhaft kinderlos bleiben werden. Für das Abfallen der Kinderlosigkeitsraten unter den Ältesten sind vor allem historische Bedingungen in der Familiengründungsphase verantwortlich. Die 72- bis 83-Jährigen des Befragungsjahres 1996 waren zur Zeit des Zweiten Weltkrieges etwa 16 bis 32 Jahre alt - also mitten in der Lebensphase, in der Partnerschaften entstehen und Familien gegründet werden. Kriegsbedingt standen aber viele junge Männer entweder nicht zur Verfügung oder kehrten aus dem Krieg nicht zurück. Die im Jahr 2014 72- bis 83-Jährigen hingegen sind die Generation der Eltern der ,Babyboomer ${ }^{c}$ - und seltener kinderlos geblieben.
Während sich der Anteil derjenigen mit Enkeln in der Altersgruppe der 42- bis 47-Jährigen im Zeitraum 1996 bis 2014 nicht verändert hat, haben die 48- bis 65-Jährigen im Jahr $2014 \mathrm{zu}$ einem geringeren Anteil Enkel als noch 1996 (Abbildung 14-1c). Beispielweise sind im Jahr 1996 noch etwa die Hälfte aller Personen im Alter von 54 bis 59 Jahren bereits Großeltern gewesen (49,0 Prozent), 18 Jahre später sind dies über zehn Prozentpunkte weniger (2014: 36,9 Prozent). Bei den über 66-Jährigen hat sich im selben Zeitraum keine Veränderung in den Anteilen derjenigen mit Enkeln gezeigt. Das Erleben der eigenen Urenkel hat sich ebenfalls zwischen 1996 und 2014 nicht verändert. Beispielhaft seien hier daher die Anteile für das Jahr 2014 genannt (ohne Abbildung): Gut jede und jeder zehnte 66- bis 71-Jährige hat Urenkel (11,0 Prozent), unter den 72- bis 77-Jährigen sind es etwa ein Viertel (23,6 Prozent) und bei den 78 - bis 83 -Jährigen schon ein gutes Drittel (34,9 Prozent). 


\section{Hohe Bildung geht für Frauen eher mit Kinderlosigkeit einher, bei Männern ist es umgekehrt.}

Insbesondere für Deutschland wird ein starker Zusammenhang zwischen dem erreichten Bildungsniveau und dauerhafter Kinderlosigkeit berichtet. Dieser Bildungsunterschied sollte in den mittleren Lebensjahren besonders deutlich sichtbar sein - nämlich dann, wenn auch unter den Hochgebildeten, die später Kinder bekommen, ein zukünftiger Übergang zur Elternschaft quasi ausgeschlossen werden kann. Hier wird exemplarisch das Jahr 2014 betrachtet und zudem jeweils Männer mit Frauen im Alter von 42 bis 65 Jahren verglichen (Abbildung 14-2). Für Personen bis 65 Jahre wurden eher abnehmende Anteile von Eltern beobachtet.

Kinderlosigkeit und Bildung hängen in der Altersgruppe der 42- bis 65-Jährigen zusam- men. Der Zusammenhang ist für Männer und Frauen allerdings gegensätzlich: Während höhere Bildung für Frauen das Risiko, kinderlos zu bleiben, erhöht, bleiben höher gebildete Männer seltener kinderlos. Dieser Befund entspricht Ergebnissen aus anderen Studien (KrätschmerHahn 2012; Dorbritz \& Schneider 2013). Eine Erklärung für diesen gegensätzlichen Bildungszusammenhang lautet wie folgt: Insbesondere hochgebildete Frauen finden selten mindestens gleich gut ausgebildete Männer oder bleiben aufgrund der hohen Schwierigkeiten bei der Vereinbarkeit von Familie und Beruf kinderlos. Anders wirkt eine hohe Bildung für Männer: Unter der Voraussetzung von Bildungsheterogamie in der Partnerwahl (das heißt hochgebildete Männer suchen eher weniger gebildete Frauen und umgekehrt) sind hochgebildete Männer auf dem Partnermarkt besonders begehrt beziehungsweise erfolgreich.

\section{Abbildung 14-2: Anteile Kinderloser (Alter 42-65 Jahre), nach Geschlecht und Bildung, im Jahr 2014 (in Prozent)}

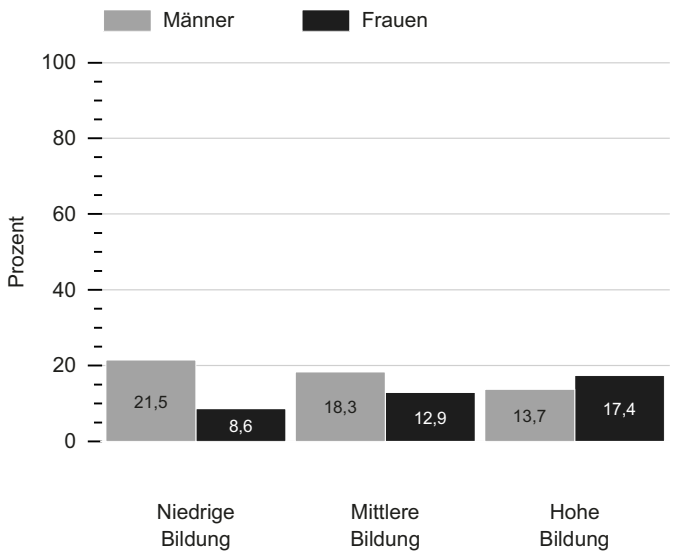

Quelle: DEAS 2014 ( $n=3.372)$, gewichtet, gerundete Angaben; $(p<, 05)$.

Die Interaktion zwischen Bildung und Geschlecht ist signifikant. Signifikante Bildungsgruppenunterschiede für Männer und Frauen in gegenläufige Richtungen. Nicht signifikant ist der Geschlechterunterschied nur bei niedriger Bildung.

Zusammenfassend lässt sich sagen, dass sich Veränderungen der familialen Generationenstrukturen einerseits in der verlängerten gemeinsamen Lebensspanne mit den Eltern oder Großeltern zeigen. Dies ist vor allem Ausdruck der gestiegenen Lebenserwartung. Die Folgen späterer Geburten oder des Ausbleibens von Elternschaft
- beides für Deutschland typische Entwicklungen - zeigen sich andererseits vor allem darin, dass im Jahr 2014 weniger Personen im mittleren Alter Eltern oder Großeltern sind. Für Menschen zu Beginn der zweiten Lebenshälfte ist es im Jahr 2014 wahrscheinlicher, noch lebende Vorfahren $\mathrm{zu}$ haben als eigene Nachkommen. 


\subsection{Wandel der Wohnentfernung zwischen Eltern und erwachsenen Kindern}

Neben dem Vorhandensein verschiedener Generationen gehört die Wohnentfernung zwischen Familienmitgliedern $\mathrm{zu}$ den strukturellen Aspekten von Generationenbeziehungen. Wie weit Eltern und Kinder voneinander entfernt wohnen, ist vor allem für die Gelegenheiten persönlicher Kontakte oder gegenseitige praktische Unterstützung im Alltag entscheidend (Hank 2015).

\section{Die Wohnentfernung zwischen Eltern und ihren erwachsenen Kindern vergrößert sich im sozialen Wandel stetig.}

Die Entwicklung einer sich vergrößernden Wohnentfernung zwischen Eltern und ihren erwachsenen Kindern außerhalb des eigenen Haushalts zeigt sich auch im Jahr 2014 (Abbildung 14-3). Während die erwachsenen Kinder im Jahr 1996 noch für 38,4 Prozent der Eltern im Mittel in der Nachbarschaft oder im gleichen Ort lebten, trifft dies im Jahr 2014 nur noch auf etwa ein Viertel (25,8 Prozent) zu. Für über die Hälfte der Eltern (51,1 Prozent) wohnen die erwachsenen Kinder im Jahr 2014 im Mittel in einem anderen Ort, aber in einem Radius von bis zu zwei Stunden Wegezeit und für ein knappes Viertel (23,1 Prozent) noch weiter entfernt.

Abbildung 14-3: Wohnentfernung zu den erwachsenen Kindern außerhalb des elterlichen Haushalts (gemittelt), in den Jahren 1996, 2002, 2008 und 2014 (in Prozent)

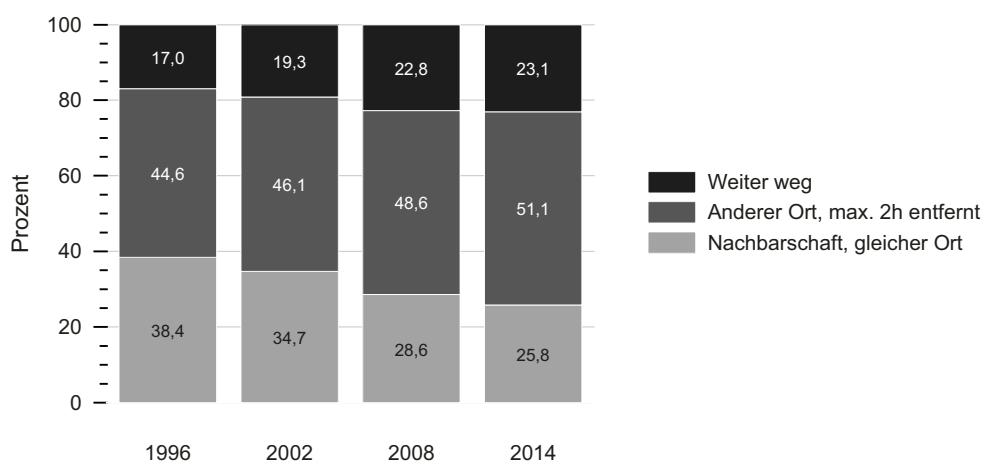

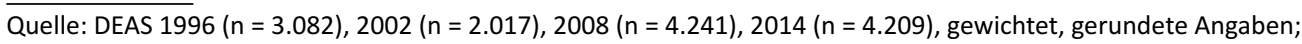
$(p<, 05)$.

Die Kategorien ,Nachbarschaft, gleicher Ort' und ,weiter weg' unterscheiden sich zwischen allen Erhebungszeitpunkten signifikant voneinander. Die Kategorie ,Anderer Ort, max. 2h entfernt' unterscheidet sich nur zwischen 1996 und 2008 bzw. 2014 statistisch signifikant, nicht jedoch zwischen 1996 und 2002 bzw. 2002 und 2008.

Neben der Betrachtung der durchschnittlichen Wohnentfernung zu allen erwachsenen Kindern ist die Frage von Bedeutung, wie weit das jeweils nächstwohnende Kind entfernt lebt. Gerade in akuten Bedarfssituationen oder Notfällen kann es entscheidend sein, wie schnell eines der Kinder vor Ort sein kann. Für die Wohnentfernung zum nächstwohnenden Kind zeigt sich über die Zeit zwischen 1996 und 2014 eine ganz ähnliche
Entwicklung (vgl. Tabelle A 14-3 im Anhang): Während das nächstwohnende Kind außerhalb des eigenen Haushalts im Jahr 1996 noch für deutlich mehr als die Hälfte der Eltern (55,0 Prozent) in der Nachbarschaft oder im selben Ort gewohnt hat, trifft dies im Jahr 2014 nur noch auf 41,5 Prozent zu. Etwa ein Drittel der Eltern (33,9 Prozent) hat im Jahr 1996 das nächste Kind im Umkreis von maximal zwei Stunden 
Wegezeit - also eine Distanz, die bei Bedarf relativ schnell überwunden werden kann, für regelmäßige persönliche Kontakte aber schon ein Hindernis darstellen kann. Im Jahr 2014 beträgt dieser Anteil schon 44,8 Prozent. Es sind im Jahr 2014 also mehr Eltern von weiteren und potenziell problematischen Wohnentfernungen zu ihren Kindern betroffen.

\section{Die Wohnentfernung wächst für jüngere Eltern stärker als für ältere.}

Insbesondere in späteren Lebensphasen, wenn zum Beispiel körperliche Einschränkungen zunehmen oder die Partnerin beziehungsweise der Partner verstirbt, ist räumliche Nähe zu den Kindern von Vorteil.

\section{Abbildung 14-4: Wohnentfernung zu den erwachsenen Kindern außerhalb des elterlichen Haushalts (gemittelt), nach Alter, in den Jahren 1996, 2002, 2008 und 2014 (in Prozent)}

Nachbarschaft, gleicher Ort

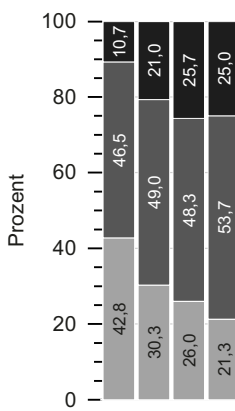

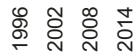

42-47

Jahre
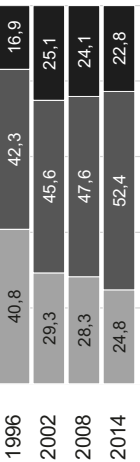

48-53

Jahre
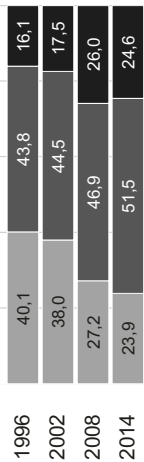

54-59

Jahre
Anderer Ort, max. $2 \mathrm{~h}$ entfernt
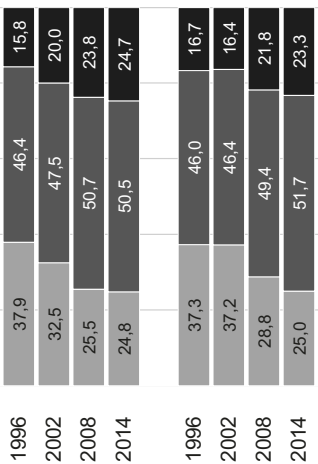

60-65

Jahre

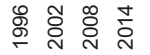

66-71

Jahre
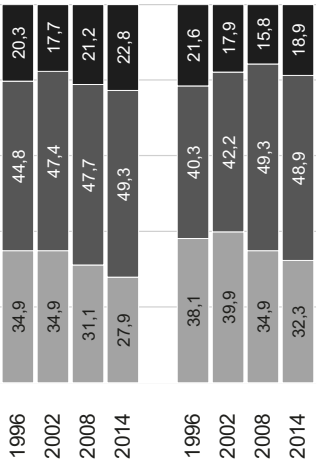

72-77

Jahre
78-83

Jahre

Quelle: DEAS $1996(n=2.999), 2002(n=1.979), 2008(n=4.141), 2014(n=4.107)$, gewichtet, gerundete Angaben; $(p<, 05)$.

Signifikante Unterschiede zwischen 1996 und 2014 auf allen Kategorien der Wohnentfernung für die unter 72-Jährigen. Für die Altersgruppe 72 bis 77 Jahre besteht zwischen 1996 und 2014 eine signifikante Veränderung nur für die Kategorie ,Nachbarschaft, gleicher Ort'. Für die Altersgruppe 78 bis 83 Jahre zeigen sich keinerlei signifikante Veränderungen.

Angesichts der allgemeinen Entwicklung hin zu größeren Wohnentfernungen zwischen Eltern und erwachsenen Kindern ist es daher wichtig zu wissen, ob dieser Wandel für alle Eltern gleichermaßen gilt oder ob bestimmte Altersgruppen besonders betroffen sind.

Im Vergleich der verschiedenen Geburtskohorten über die Zeit (Abbildung 14-4) zeigt sich, dass die Entwicklung der mittleren Wohnentfernung altersgruppenspezifisch ist: Von der allgemeinen Vergrößerung der Wohnentfernung über die Zeit sind die jüngeren Eltern stärker betroffen. Beispielweise hat sich der Anteil der Eltern im Alter von 42 bis 47 Jahren, für die die Kinder im Mittel mindestens im selben Ort wohnen, im Zeitraum zwischen 1996 und 2014 halbiert (1996: 42,8 Prozent; 2014: 21,3 Prozent). In der Altersgruppe 66 bis 71 Jahre fällt diese Veränderung schon deutlich geringer aus (Nachbarschaft, gleicher Ort 1996: 37,3 Prozent; 2014: 25,0 Prozent) und unter den hier betrachteten ältesten Eltern (78 bis 83 Jahre) zeigt sich keinerlei Veränderung der mittleren Wohnentfernung zu den Kindern. Anders ausgedrückt: Während sich die mittlere Wohnentfernung zu den erwachsenen Kindern im Jahr 1996 noch für alle Altersgruppen sehr ähnlich dargestellt hat, zeigen sich im Jahr 2014 deutliche Unterschiede. Auch bei der Betrach- 
tung des nächstwohnenden erwachsenen Kindes außerhalb des Haushalts zeigt sich eine weniger deutliche Vergrößerung der Wohnentfernungen bei den älteren Eltern: Für die jüngeren Eltern (42 bis 47 Jahre) verringert sich der Anteil derjenigen mit dem nächsten Kind im selben Ort im Zeitraum von 1996 bis 2014 um etwa 20 Prozentpunkte, bei den 78- bis 83-jährigen Eltern beträgt die Verringerung nur acht Prozentpunkte (ohne Abbildung).

Insofern sind glücklicherweise gerade diejenigen Eltern, die vermutlich in einem höheren Maße Bedarf an Unterstützung im Alltag haben, von der allgemeinen Entwicklung der größer werdenden Wohnentfernungen weniger betroffen. Allerdings steht zu befürchten, dass sich diese Lage verändern wird, wenn nachfolgende Geburtskohorten das hohe Erwachsenenalter erreichen.

\section{Insbesondere hochgebildete Eltern haben weiter entfernt wohnende erwachsene Kinder.}

Ein wichtiger Einflussfaktor auf die Wohnentfernung ist zu jedem Zeitpunkt zwischen 1996 und 2014 die Bildung. Exemplarisch für 2014 zeigt sich, dass mit höherer Bildung des Elternteils auch eine größere mittlere Wohnentfernung zu den erwachsenen Kindern einhergeht (Abbildung 14-5). Die mittlere Wohnentfernung liegt für ein gutes Drittel der Eltern mit niedriger Bildung (35,6 Prozent) in der Nachbarschaft oder im gleichen Ort - unter hochgebildeten Eltern ist dies nur für etwa ein Fünftel der Fall (19,9 Prozent).

Abbildung 14-5: Wohnentfernung zu den erwachsenen Kindern außerhalb des elterlichen Haushalts (gemittelt), nach Bildung, im Jahr 2014 (in Prozent)

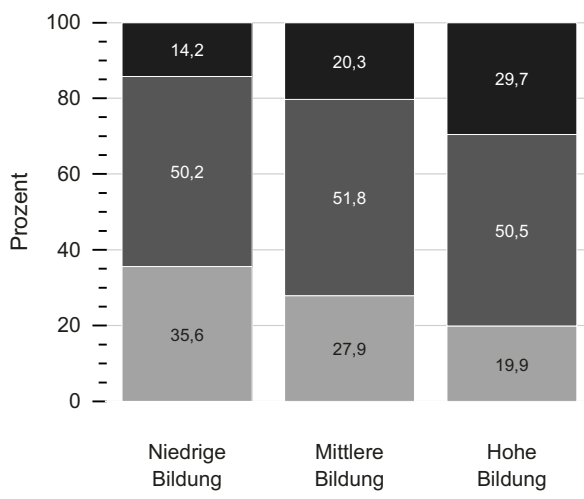

Weiter weg

Anderer Ort, max. $2 \mathrm{~h}$ entfernt

Nachbarschaft, gleicher Ort

Quelle: DEAS 2014 ( $n=4.209)$, gewichtet, gerundete Angaben; $(p<, 05)$.

Signifikante Bildungsgruppenunterschiede für alle Kategorien außer ,Anderer Ort, max. $2 \mathrm{~h}$ entfernt”

Gleichzeitig ist der Anteil von Eltern mit einer mittleren Wohnentfernung von mehr als zwei Stunden Wegezeit (,weiter weg') unter Eltern mit hoher Bildung etwa doppelt so groß $(29,7$ Prozent) wie unter Eltern mit niedriger Bildung (14,2 Prozent). Betrachtet man wiederum die Distanz zum nächstwohnenden Kind, so haben deutlich mehr als die Hälfte der Eltern mit niedriger Bildung (55,6 Prozent) im Jahr 2014 das nächste Kind in der Nachbarschaft oder im selben Ort, aber nur etwa ein Drittel der hochgebildeten Eltern (35,1 Prozent) (ohne Abbildung). Begründet werden können diese Bildungseffekte mit der größeren Mobilität unter höher Gebildeten. Die Kinder ziehen zum Studium oder für eine Berufstätigkeit häufiger weiter weg. Höher gebildete Eltern sind in Bezug auf die ,Verfügbarkeit' nah wohnender Kinder also im Nachteil. Sie sind aber auch diejenigen, die Hilfe im Alltag, die von den Kindern aufgrund der grö- 
ßeren Entfernung möglicherweise nicht geleistet werden kann, besser kompensieren können (zum Beispiel durch den Einkauf von Hilfen) beziehungsweise sie aufgrund ihrer besseren gesundheitlichen Situation (vgl. Kapitel 8) gar nicht erst so stark benötigen wie Eltern mit geringerer Bildung (Schöllgen, Huxhold, Schüz, \& Tesch-Römer 2011).

\subsection{Wandel der Kontakthäufigkeit und der Beziehungsenge zwischen Eltern und erwachsenen Kindern}

Die Bewertung der Familienbeziehungen im Allgemeinen ist über den gesamten Zeitraum von 1996 bis 2014 stabil hoch geblieben. $\mathrm{Zu}$ allen Zeitpunkten zwischen 1996 und $2014 \mathrm{emp}-$ finden mehr als 77 Prozent der 40- bis 85-Jährigen ihre Familienbeziehungen insgesamt als gut oder sehr gut. Höchstens fünf Prozent beurteilen sie als schlecht oder sehr schlecht. Dabei berichten Ältere und Frauen durchgängig eine etwas bessere Bewertung ihrer Familienbeziehungen (ohne Abbildung). Im Folgenden werden spezifischere Aspekte der Beziehungen zu den erwachsenen Kindern untersucht - die Häufigkeit des Kontaktes und die Beziehungsenge.

\section{Die Kontakthäufigkeit und die} Beziehungsenge zwischen Eltern und erwachsenen Kindern bleiben stabil hoch.
Ein ganz anderes Bild als für die Veränderung der Wohnentfernungen zeigt sich für die Kontakthäufigkeit und die emotionale Nähe zu den erwachsenen Kindern. Beide sind über den gesamten Zeitraum von 1996 bis 2014 stabil hoch geblieben.

Mehr als 78 Prozent der Eltern haben zu jedem Zeitpunkt zwischen 1996 und 2014 mindestens wöchentlich Kontakt zu ihren Kindern außerhalb des Haushalts. Weniger als jeder zehnte Elternteil hat seltener als monatlich Kontakt $\mathrm{zu}$ den Kindern (Abbildung 14-6a). Auch die Beziehungsenge zu den erwachsenen Kindern ist im Zeitraum 1996 bis 2014 stabil hoch geblieben: Mehr als 88 Prozent der Eltern berichten $\mathrm{zu}$ jedem Zeitpunkt eine enge oder sehr enge Verbundenheit mit ihren Kindern außerhalb des Haushalts. Höchstens vier Prozent berichten von nicht engen Beziehungen (Abbildung 14-6b).

Abbildung 14-6: Kontakthäufigkeit und Beziehungsenge zu den erwachsenen Kindern außerhalb des elterlichen Haushalts (jeweils gemittelt), in den Jahren 1996, 2002, 2008 und 2014 (in Prozent)

a) Kontakthäufigkeit






\section{b) Beziehungsenge}

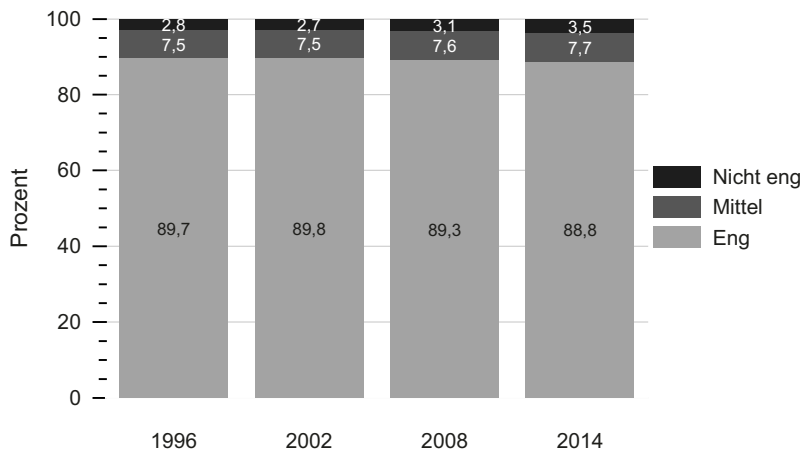

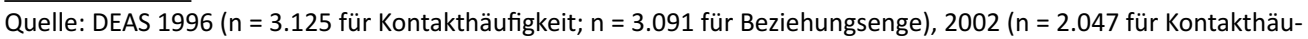
figkeit; $n=2.030$ für Beziehungsenge), 2008 ( $n=4.199$ für Kontakthäufigkeit und Beziehungsenge), 2014 ( $n=4.166$ für Kontakthäufigkeit; $\mathrm{n}=4.163$ für Beziehungsenge), gewichtet, gerundete Angaben, $(p<, 05)$.

Es finden sich keinerlei statistisch signifikante Veränderungen der mittleren Kontakthäufigkeit oder der mittleren Beziehungsenge über die Zeitpunkte.

Es zeigen sich durchgängig zu allen Zeitpunkten zwischen 1996 und 2014 Geschlechtsunterschiede: Väter haben seltener Kontakt zu ihren Kindern als Mütter. Beispielsweise berichten im Jahr 2014 72,8 Prozent der Väter einen mindestens wöchentlichen Kontakt, unter den Müttern sind es 84,0 Prozent (vgl. Tabelle A 14-4 im Anhang). Entgegen der Vermutung sich annähernder Beziehungsmuster zwischen Müttern und Vätern vergrößern sich die Geschlechtsunterschiede im Kontakt sogar geringfügig über die Zeit. Möglicherweise gehen Mütter und Väter mit größeren Wohnentfernungen unterschiedlich um. Vielleicht gelingt es Müttern besser, auch über größere Distanzen hinweg regelmäßig Kontakt zu halten, als dies für Väter der Fall ist und weichen leichter auf andere Arten des Austausches als persönliche Treffen aus (Treas \& Gubernskaya 2012). Ähnlich der Kontakthäufigkeit unterscheiden sich Mütter und Väter auch zu jedem Zeitpunkt in der Verbundenheit zu ihren Kindern. Mütter fühlen sich enger verbunden. Im Jahr 2014 haben 92,3 Prozent der Mütter enge oder sehr enge Beziehungen, unter den Vätern sind es 84,7 Prozent (vgl. Tabelle A 14-5 im Anhang). Diese Geschlechtsunterschiede haben sich über die Zeit nicht verändert. Unterschiede zwischen den Altersgruppen sind sehr gering und zeigen sich auch nur für die Beziehungsenge. Im Jahr 2014 fühlen sich Eltern im Alter von 40 bis 54 Jahren mit ihren Kindern etwas weniger verbunden als ältere Eltern: Unter den 40- bis 54-Jährigen berichten 87,4 Prozent (sehr) enge Beziehungen, unter den Älteren sind es 89,3 beziehungsweise 89,1 Prozent (vgl. Tabelle A 14-5 im Anhang). Es bestehen seit 2008 geringe Unterschiede zwischen den Bildungsgruppen in der Art, dass höher gebildete Eltern einen etwas häufigeren Kontakt und etwas engere Beziehungen zu den Kindern berichten (ohne Abbildung). 


\subsection{Häufigkeit der Gefühle von Ärger und Wut in den Beziehungen zu erwachsenen Kindern}

Erstmals kann der DEAS für das Jahr 2014 auch als eher negativ assoziierte Beziehungsaspekte zwischen Eltern und Kindern darstellen. Im Folgenden geht es darum, wie häufig Eltern und Kinder Wut und Ärger füreinander empfinden.

\section{Gefühle wie Wut und Ärger sind in den Beziehungen zwischen Eltern und ihren erwachsenen Kindern selten.}

Auf die Frage, wie häufig Eltern und Kinder wütend oder ärgerlich aufeinander sind, antwortet die Mehrheit aller Eltern (77,1 Prozent), dass dies nur selten oder gar nicht der Fall ist (Abbildung 14-7). Dieses Ergebnis passt zu den bereits berichteten Ergebnissen und belegt die insgesamt hohe Qualität der Eltern-Kind-Beziehungen in der zweiten Lebenshälfte.

Abbildung 14-7: Häufigkeit von Wut und Ärger in den Beziehungen zu den erwachsenen Kindern außerhalb des elterlichen Haushalts (gemittelt), gesamt, nach Alter und Geschlecht, im Jahr 2014 (in Prozent)

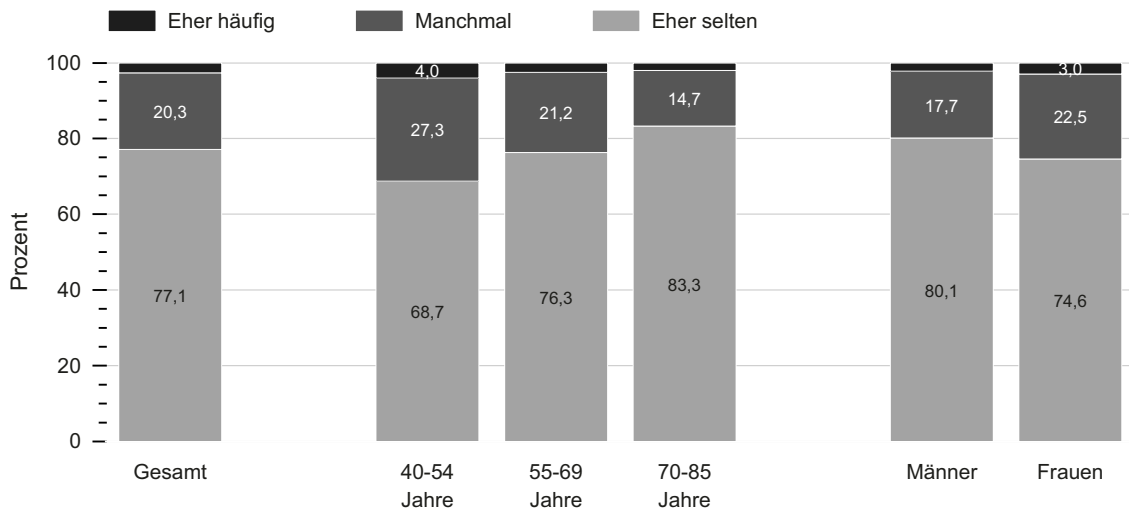

Quelle: DEAS $2014(n=4.134)$, gewichtet, gerundete Angaben, $(p<, 05)$.

Alle Altersgruppenunterschiede sind signifikant außer der Unterschied in der Kategorie ,eher häufig' zwischen den Altersgruppen 55 bis 69 und 70 bis 85 Jahre. Alle Geschlechtsunterschiede sind signifikant außer der in der Kategorie ,eher häufig'.

Aber insgesamt fast ein Viertel der Eltern gibt an, manchmal (20,3 Prozent) oder eher häufig (2,7 Prozent) Wut und Ärger gegenüber den Kindern $\mathrm{zu}$ empfinden. Geht man davon aus, dass Eltern eher ungern negative Gefühle für ihre Kinder berichten - und somit Wut und Ärger vermutlich häufiger auftreten als angegeben - deuten diese Zahlen auf ein nicht unerhebliches Konfliktpotenzial zwischen Eltern und Kindern hin. Dabei müssen Wut und Ärger nicht ausschließlich schlecht für diese Beziehungen sein, sondern können auch konstruktiv Veränderungen herbeiführen.
Ältere Eltern haben seltener Gefühle von Wut und Ärger als jüngere Eltern (Abbildung 14-7). Knapp ein Drittel der Eltern zwischen 40 und 54 Jahren gibt an, mindestens manchmal wütend und ärgerlich auf die eigenen Kinder zu sein (manchmal: 27,3 Prozent; eher häufig: 4,0 Prozent). Bei den ältesten Eltern (70 bis 85 Jahre) ist dieser Anteil deutlich geringer (manchmal: 14,7 Prozent; eher häufig: 2,0 Prozent). Vermutlich sind Ablösungsprozesse (Postadoleszenz) mitverantwortlich dafür, dass es in den jüngeren Altersgruppen häufiger solche Gefühle gibt. Außerdem zeigen ältere Menschen häufiger als 
jüngere die Tendenz, Konflikte zu vermeiden (Charles 2010). Im Gegensatz zur Kontakthäufigkeit und zur emotionalen Nähe zeigen sich für Wut und Ärger Geschlechtsunterschiede in die andere Richtung (Abbildung 14-7): Mütter haben insgesamt häufiger Gefühle von Wut und Ärger. Zunächst scheint dieses Ergebnis etwas paradox, da erwartet werden könnte, dass Mütter aufgrund ihrer in der Regel engeren
Beziehungen insgesamt weniger Konflikte berichten als Väter. Aber Mütter haben durch ihre engeren und kontaktintensiveren Beziehungen möglicherweise auch mehr Gelegenheit zu konflikthaften Begegnungen und gehen diesen zudem weniger aus dem Weg, als dies Väter vielleicht tun (Salzburger 2014). Unterschiede zwischen Bildungsgruppen bestehen nicht (ohne Abbildung).

\subsection{Diskussion und Implikationen}

Die generationalen Strukturen, in denen sich Familienbeziehungen in der zweiten Lebenshälfte abspielen, sind im Wandel begriffen. Bedingt durch die steigende Lebenserwartung können Menschen im Jahr 2014 bis in ihr sechstes Lebensjahrzehnt hinein zu einem beachtlichen Teil von etwa einem Drittel noch ihre eigenen Eltern erleben. Sogar Großeltern hat zu Beginn der zweiten Lebenshälfte noch etwa jede zehnte Person. Ein anderes Bild hingegen zeigt sich für die nachfolgenden Familiengenerationen: Jüngere Geburtskohorten bleiben häufiger kinderlos und auch Enkel werden seltener geboren. Die familialen Strukturen werden also einerseits nach ,oben' länger, nach ,unten' aber kürzer und dünner. Mit Blick auf die Zukunft bedeutet dies, dass auf Menschen in der zweiten Lebenshälfte möglicherweise gehäuft Sorgeaufgaben für ältere Familiengenerationen zukommen. Gleichzeitig könnten jüngere Familienmitglieder aber immer seltener für die Übernahme von Unterstützungsleistungen zur Verfügung stehen. Dementgegen steht im Jahr 2014 eine insgesamt sehr hohe Qualität der gelebten Beziehungen: Ältere Eltern und ihre erwachsenen Kinder haben häufig Kontakt, sie stehen sich emotional nah und haben eher selten Konflikte.

Im sozialen Wandel wachsen die Wohnentfernungen zwischen älteren Eltern und ihren erwachsenen Kindern jedoch stetig. Die Strukturen von familialen Beziehungen sind also in mehrfacher Hinsicht im Wandel begriffen. Doch wenn aus dem Wandel von Strukturen kurzerhand Schlüsse auf die Qualität der Beziehungen zwischen älteren Eltern und ihren erwachsenen Kindern gezogen werden, sollte dies mit Bedacht und in einer differenzierten Art und Weise geschehen. Offensichtlich bleiben Verbundenheit und Kontakt zu den Kindern auf einem hohen Niveau stabil - auch bei einer stetigen Vergrößerung der Wohnentfernungen. Veränderte Kommunikationsformen, zum Beispiel Videotelefonie oder soziale Netzwerke, können eine Beziehungspflege auch über große räumliche Distanzen erleichtern. Insofern ist davon auszugehen, dass sich Veränderungen der familialen Strukturen unterschiedlich auf verschiedene Aspekte der Ausgestaltung von Familienbeziehungen auswirken. $\mathrm{Ob}$ und inwiefern sich zwischen älteren Eltern und ihren erwachsenen Kindern der Austausch von Hilfen im Alltag verändert, die räumliche Nähe voraussetzen, wird in Kapitel 16 dieses Buches näher behandelt.

Das Bildungsniveau spielt eine Rolle für die Generationenbeziehungen - und das nicht nur für das Vorhandensein familialer Generationen, sondern auch für die räumlichen Strukturen. So lassen sich bereits bekannte Zusammenhänge zwischen Bildung und Kinderlosigkeit mit den DEAS-Daten bestätigen. Auch die Ergebnisse zu weiteren Wohnentfernungen bei höher Gebildeten sind konform mit der bestehenden Literatur (Hank 2007). Eltern, die über ein hohes Bildungsniveau verfügen, haben ganz ähnlich kontaktintensive und emotional enge Beziehungen zu ihren Kindern wie geringer gebildete Eltern. Und dies, obwohl gerade hochgebildete Eltern häufiger in größerer räumlicher Distanz zu ihren 
Kindern leben. Dies ist eventuell Ausdruck von Ressourcen, die helfen, mit strukturellen Veränderungen wie wachsenden Wohnentfernungen gut umgehen zu können. Zusammengefasst lässt sich sagen: Während höher Gebildete zwar eher von Kinder- und Enkellosigkeit sowie von kleineren und räumlich gestreuten Familiennetzen betroffen sind, scheinen sie in Bezug auf die allgemeine Qualität der Generationenbeziehungen wiederum keine Nachteile zu erfahren.

Zudem sind familiale Beziehungen oftmals von Vereinbarkeitsproblematiken $\mathrm{zu}$ anderen Lebensbereichen betroffen. So kann die Sorge für ältere, gesundheitlich eingeschränkte Eltern oder die Betreuung von (Enkel-)Kin- dern mit Anforderungen aus dem beruflichen Alltag in Konflikt geraten (vgl. Kapitel 12 und 15). Angesichts des Wandels von generationalen und räumlichen Strukturen wird es für die Aufrechterhaltung von Lebensqualität im Alter auch von Bedeutung sein, dass familiale Unterstützungsleistungen möglichst flexibel mit einer Erwerbstätigkeit vereinbar sind. Das Gesetz zur besseren Vereinbarkeit von Familie, Pflege und Beruf greift diese Lebenssituationen auf. Zum anderen wird es wichtig sein, dass ältere Menschen - insbesondere im Falle von Kinderlosigkeit - verlässliche Unterstützungsstrukturen auch jenseits von Verwandtschaft etablieren können (vgl. Kapitel 17).

\section{Literatur}

Bertram, H. (2000). Die verborgenen familiären Beziehungen in Deutschland: Die multilokale Mehrgenerationenfamilie. In: M. Kohli \& M. Szydlik (Hrsg.) Generationen in Familie und Gesellschaft (S. 97-121). Opladen: Leske+Budrich.

Charles, S. T. (2010). Strength and vulnerability integration: A model of emotional well-being across adulthood. Psychological Bulletin, 136(6), 1068-1091. doi: 10.1037/a0021232.

Dorbritz, J., \& Schneider, N. F. (2013). Familiendemografische Trends in Deutschland - Herausforderungen für zukünftiges politisches Handeln In: M. Hüther \& G. Naegele (Hrsg.) Demografiepolitik (S. 142-163). Wiesbaden: Springer VS.

Hank, K. (2015). Intergenerationale Beziehungen. In: P. B. Hill \& J. Kopp (Hrsg.) Handbuch Familiensoziologie (S. 463-486). Wiesbaden: Springer VS.

Hank, K. (2007). Proximity and contacts between older parents and their children: A European comparison. Journal of Marriage and Family, 69, 157-173.

Huxhold, O., Mahne, K., \& Naumann, D. (2010). Soziale Integration. In: A. Motel-Klingebiel, S. Wurm \& C. Tesch-Römer (Hrsg.) Altern im Wandel. Befunde des Deutschen Alterssurveys (DEAS) (S. 215-233). Stuttgart: Kohlhammer.

Krätschmer-Hahn, R. (Hrsg.). (2012). Kinderlosigkeit in Deutschland. Zum Verhältnis von Fertilität und Sozialstruktur. Wiesbaden: VS Verlag für Sozialwissenschaften.
Mahne, K., \& Motel-Klingebiel, A. (2010). Familiale Generationenbeziehungen. In: A. Motel-Klingebiel, S. Wurm \& C. Tesch-Römer (Hrsg.) Altern im Wandel. Befunde des Deutschen Alterssurveys (DEAS) (S. 188214). Stuttgart: Kohlhammer.

Peuckert, R. (2012). Familienformen im sozialen Wandel (8., vollst. überarb. Aufl. Aufl.). Wiesbaden: VS Verlag für Sozialwissenschaften.

Rosenmayr, L., \& Köckeis, E. (1963). Propositions for a Sociological Theory of Ageing and the Family. International Social Science Journal, XV, 410-426.

Rossi, P. H., \& Rossi, A. S. (1990). Of human bonding: Parent-child relations across the life course. New York: Aldine de Gruyter.

Salzburger, V. (2014). Die Geburt des ersten Enkelkindes: Zur Adaption von Generationenbeziehungen. Wiesbaden: Springer VS.

Schöllgen, I., Huxhold, O., Schüz, B., \& Tesch-Römer, C. (2011). Resources for health: Differential effects of optimistic self-beliefs and social support according to socioeconomic status. Health Psychology, 30(3), 326335. doi: $10.1037 / \mathrm{a} 0022514$.

Treas, J., \& Gubernskaya, Z. (2012). Farewell to moms? Maternal contact for seven countries in 1986 and 2001. Journal of Marriage and Family, 74(2), 297-311. doi: 10.1111/j.1741-3737.2012.00956.x.

Open Access Dieses Kapitel wird unter der Creative Commons Namensnennung 2.5 International Lizenz (http://creativecommons.org/licenses/by/2.5/deed.de) veröffentlicht, welche die Nutzung, Vervielfältigung, Bearbeitung, Verbreitung und Wiedergabe in jeglichem Medium und Format erlaubt, sofern Sie den/die ursprünglichen Autor(en) und die Quelle ordnungsgemäß nennen, einen Link zur Creative Commons Lizenz beifügen und angeben, ob Änderungen vorgenommen wurden.

Die in diesem Kapitel enthaltenen Bilder und sonstiges Drittmaterial unterliegen ebenfalls der genannten Creative Commons Lizenz, sofern sich aus der Abbildungslegende nichts anderes ergibt. Sofern das betreffende Material nicht unter der genannten Creative Commons Lizenz steht und die betreffende Handlung nicht nach gesetzlichen Vorschriften erlaubt ist, ist für die oben aufgeführten Weiterverwendungen des Materials die Einwilligung des jeweiligen Rechteinhabers einzuholen. 